\section{ORIGINAL RESEARCH}

K. Aydin

O. Okur

B. Tatli

S.G. Sarwar

C. Ozturk

C. Dilber

\title{
Reduced Gray Matter Volume in the Frontotemporal Cortex of Patients with Early Subacute Sclerosing Panencephalitis
}

\begin{abstract}
BACKGROUND AND PURPOSE: Subacute sclerosing panencephalitis (SSPE) is a persistent infection of the central nervous system by the measles virus. Patients in the initial stages of SSPE show behavioral symptoms and usually normal cranial MR imaging findings. We aimed to investigate the gray matter volume changes in patients with early SSPE.
\end{abstract}

\begin{abstract}
MATERIALS AND METHODS: Seventeen patients with SSPE with normal cranial MR imaging findings and 30 sex- and age-matched control subjects were included in the study. Clinical parameters of the patients were quantified by using a neurologic disability index (NDI) as defined previously. We obtained T1-weighted magnetization-prepared rapid acquisition gradient echo images from the patients and control subjects, and we applied an optimized method of voxel-based morphometry. We performed a cross-sectional analysis to search the gray matter volume differences between the patients and control subjects. The correlation between the gray matter distribution and the duration of symptoms, immunoglobulin G index, and NDI scores was tested.
\end{abstract}

\begin{abstract}
RESULTS: We found that the cortical gray matter volume of patients was reduced in the frontotemporal regions including the bilateral cingulate cortex and amygdala. There was no correlation between the gray matter distribution of patients and the duration of symptoms or the NDI scores.
\end{abstract}

CoNCLUSIONS: The current study demonstrated gray matter volume reduction in the frontotemporal cortex of patients with SSPE without any apparent lesions on conventional MR imaging. Because the cingulate cortex and amygdala are involved in emotion processing, gray matter loss in these regions may contribute to the development of early behavioral symptoms of SSPE.

$S^{3}$ ubacute sclerosing panencephalitis (SSPE) is a slow virus infection of the central nervous system, which is caused by the measles virus. ${ }^{1}$ It is a rare infectious disease in developed countries. An incidence of 1 case per million patients with measles infection a year was reported in developed countries. ${ }^{1}$ However, it is still prevalent in underdeveloped and developing countries. ${ }^{2}$ The characteristic clinical presentation of SSPE usually starts with nonspecific cognitive decline and behavioral changes, such as memory impairments, decreased scholastic performance, reduction of social interactions, and emotional instability. ${ }^{1}$ Following the behavioral changes, involuntary movements, such as myoclonic jerks and choreoathetosis, develop. Myoclonic jerks initially affect the head and then involve the trunk and limbs with progression of disease. Spasticity, visual disturbances, and language difficulties usually accompany the involuntary movements. In late stages of the disease, mental deterioration evolves, spasticity progresses into opisthotonus, and terminal patients pass into a vegetative state.

T2-weighted hyperintense signal-intensity changes in the

Received July 24, 2008; accepted after revision August 15.

From the Departments of Neuroradiology (K.A.), Pediatric Neurology (B.T.), and Radiology (S.G.S.), Istanbul Faculty of Medicine, Istanbul University, Istanbul, Turkey; Institute of Biomedical Engineering (0.0., C.0.), Bogazici University, Bebek, Istanbul, Turkey; and Department of Pediatrics (C.D.), Kahramanmaras Sutcu Imam University, Kahramanmaras, Turkey.

This work was supported by the Outstanding Young Scientist Award Grant of the Turkish Academy of Sciences (GEBIP-TUBA).

Please address correspondence to Kubilay Aydin, MD, Adnan Saygun Caddesi, M.Salih Rustu bey sokak, Ulus Konaklari, No. 8/12, Ulus, Istanbul, Turkey; e-mail: draydink@ superonline.com or aydink@istanbul.edu.tr

Indicates open access to non-subscribers at www.ajnr.org

DOI 10.3174/ajnr.A1329 cerebral cortex, periventricular white matter, basal ganglia, and brain stem and atrophic dilation of cerebral sulci are the cranial MR imaging findings of SSPE that frequently develop in the late stages of the disease. ${ }^{3-7}$ Conventional cranial imaging performed in the initial stages of SSPE usually does not reveal any pathology. However, recent MR spectroscopy studies have demonstrated that the metabolic alterations caused by neuronal loss and gliosis were present even in normalappearing cerebral tissue in the initial stages of SSPE. ${ }^{8,9}$ Voxelbased morphometry is an automated statistical image-analysis method that is used to search gray matter volume differences between various subject groups. ${ }^{10}$ Voxel-based morphometry can detect minor morphologic changes that could not be detected by visual case analyses.

The purpose of the current study was to investigate the gray matter volume changes in the initial stages of SSPE. We studied patients with a diagnosis of SSPE whose conventional cranial MR imaging findings appeared normal. We hypothesized that neuronal degeneration/loss starting in the early stages of SSPE would cause cerebral gray matter loss that could be detected by using voxel-based morphometry. We performed a cross-sectional voxel-based morphometry study to search the gray matter volume differences between the patients with SSPE and age- and sex-matched control subjects. We also studied the correlations between the gray matter distribution and the clinical variables.

\section{Materials and Methods}

\section{Patients}

We studied 22 patients with a diagnosis of SSPE. Because cerebral parenchymal lesions would cause segmentation errors during image 


\begin{tabular}{|c|c|c|c|c|c|c|c|}
\hline $\begin{array}{l}\text { Patient } \\
\text { No. }\end{array}$ & Age & Sex & $\begin{array}{l}\text { Duration of Symptoms } \\
\text { (weeks) }\end{array}$ & Stage & $\begin{array}{l}\text { NDI } \\
\text { Score }\end{array}$ & $\lg G$ Index & Symptoms \\
\hline 1 & 10 & $M$ & 8 & 1 & 10 & 37 & $A L P, D S P$ \\
\hline 2 & 12 & $\mathrm{~F}$ & 3 & 1 & 15 & 37 & DSP, DSI, El \\
\hline 3 & 7 & $\mathrm{~F}$ & 16 & 2 & 20 & 99 & Moderate depression, DSI, INS \\
\hline 4 & 14 & $\mathrm{~F}$ & 8 & 1 & 10 & 73 & ALP, DSP \\
\hline 5 & 9 & $\mathrm{~F}$ & 5 & 1 & 10 & 113 & DSI, El \\
\hline 6 & 7 & $\mathrm{~F}$ & 24 & 2 & 25 & 108 & ALP, DSP, DSI, INS \\
\hline 7 & 8 & $\mathrm{~F}$ & 12 & 2 & 15 & 433 & DSP, INS \\
\hline 8 & 13 & $\mathrm{~F}$ & 8 & 1 & 15 & 17 & IPE, El, DSP \\
\hline 9 & 10 & M & 6 & 1 & 10 & 13 & ALP, DSI \\
\hline 10 & 9 & $\mathrm{~F}$ & 6 & 1 & 15 & 105 & El, IPE \\
\hline 11 & 10 & $\mathrm{~F}$ & 4 & 1 & 15 & 31 & ALP, DSP, DSI, EI \\
\hline 12 & 7 & M & 5 & 1 & 15 & 709 & DSI, IPE \\
\hline 13 & 13 & $\mathrm{~F}$ & 18 & 2 & 25 & 22 & ALP, DSP, DSI, INS \\
\hline 14 & 7 & M & 8 & 1 & 20 & 161 & DSP, El, IPE \\
\hline 15 & 9 & M & 12 & 2 & 25 & 47 & DSP, INS \\
\hline 16 & 9 & $\mathrm{~F}$ & 4 & 1 & 15 & 1370 & ALP, DSP, DSI, IPE \\
\hline 17 & 9 & $M$ & 5 & 1 & 10 & 126 & DSP, IPE \\
\hline
\end{tabular}

Note:-ALP indicates altered personality/behavior; DSP, decreased scholastic performance; DSI, decreased social interactions; El, emotional instability; IPE, inappropriate emotions; INS, involuntary spasms; NDI, neurologic disability index; IgG, immunoglobulin G.

analysis, we excluded 5 patients whose conventional cranial MR images revealed cortical or white matter lesions. Thus, 17 patients (11 girls and 6 boys) were included into the study. The mean age of the patients was $9.5 \pm 2.2$ years (range, $7-14$ years). Table 1 summarizes the demographic data and the clinical findings of these patients. The diagnosis of SSPE was based on the presence of clinical symptoms, typical electroencephalography findings (bilateral periodic high-amplitude slow wave bursts), and high antimeasles antibody titers in serum $(>1: 180)$ and CSF $(>1: 4){ }^{1,11}$ The mean age of initial measles infection was 28 months (ranging between 8 and 45 months).

We used the clinical staging of patients as defined by Jabbour et al. ${ }^{11}$ Briefly, stage 1 indicated behavioral changes; stage 2 , the presence of myoclonus, incoordination, choreoathetosis, and motor convulsions; stage 3, opisthotonus, decerebrate rigidity, and coma; and stage 4 , diminished rigidity, less frequent myoclonus, and loss of cerebral cortical function. Clinical parameters were also quantified by using a neurologic disability index (NDI) as defined previously for patients with SSPE by Dyken et al, ${ }^{12}$ in which motor, sensory, and mental states were evaluated; higher scores indicated poorer neurologic status. Neurologic examinations, clinical staging, and NDI scoring were performed by an experienced pediatric neurologist (B.T.). All patients were treated with oral isoprinosine (dosage, $100 \mathrm{mg} / \mathrm{kg} /$ day).

The patients were compared with a control group composed of 30 healthy subjects (20 girls and 10 boys). The mean age of the control subjects was $9.3 \pm 2.4$ years (range, $7-14$ years). None of the control subjects had a history of any neurologic or psychiatric disorder. The exclusion criteria for the patient and control groups also included any contraindication for MR imaging, a history of previous head trauma leading to hospitalization, and a previous history of febrile or nonfebrile convulsion. Written informed consent was obtained from the parents of the patients and control subjects. The study was approved by the local human subject committee.

\section{Image Acquisition}

Cranial MR imaging studies were performed on a $1.5 \mathrm{~T}$ superconducting whole-body MR imaging system (Symphony Maestro; Siemens Medical Systems, Erlangen, Germany) with a standard quadrature head coil. Conventional cranial MR imaging was performed to search for any pathologic finding that would lead to the exclusion of patients from the study. MR images were evaluated by an experienced neuroradiologist who was blinded to whether images were from a patient or control subject. The conventional cranial MR imaging protocol included axial fluid-attenuated inversion recovery (FLAIR) $(\mathrm{TR}=9800$ $\mathrm{ms}, \mathrm{TE}=110 \mathrm{~ms}, \mathrm{TI}=2100 \mathrm{~ms}, \mathrm{NEX}=2)$, axial T1-weighted spinecho $(\mathrm{TR}=500 \mathrm{~ms}, \mathrm{TE}=18 \mathrm{~ms}, \mathrm{NEX}=3)$, and coronal T2-weighted fast spin-echo $(\mathrm{TR}=9200 \mathrm{~ms}, \mathrm{TE}=110 \mathrm{~ms}, \mathrm{NEX}=3$ ) sequences. For voxel-based morphometry, high-resolution anatomic images of the whole brain were acquired from the patients and control subjects with T1-weighted magnetization-prepared rapid acquisition gradient echo sequences $(\mathrm{TR}=11.08 \mathrm{~ms}, \mathrm{TE}=4 \mathrm{~ms}, \mathrm{TI}=300 \mathrm{~ms}$, relaxation delay time $=500 \mathrm{~ms}$, flip angle $=15^{\circ}, \mathrm{FOV}=256 \times 192 \mathrm{~mm}$, matrix size $=256 \times 192$ ), yielding 128 sagittal sections with a defined voxel size of $1 \times 1 \times 1.3 \mathrm{~mm}$.

\section{Voxel-Based Morphometry Protocol and Data Preprocessing}

We applied an optimized method of voxel-based morphometry by using SPM2 (http://www.fil.ion.ucl.ac.uk/spm/software/spm2; Wellcome Department of Cognitive Neurology, London, UK) running under Matlab (MathWorks, Natick, Mass). ${ }^{10,13}$ Preprocessing of the data involved spatial normalization of all images into a standardized anatomic space, segmentation into gray and white matter, modulation, and spatial smoothing with a Gaussian kernel. T1-weighted MR images were normalized to the standard T1 template of the Montreal Neurologic Institute (MNI). The normalized images were smoothed and averaged to obtain a study-specific T1 template. All of the original structural MR images in native space were then normalized to this study-specific template. The normalized images were segmented into CSF, gray matter, and white matter compartments by using the SPM2 priors. Afterward, CSF, gray matter, and white matter images were smoothened with an 8-mm full width at half maximum (FWHM) kernel and averaged to obtain study-specific CSF, gray matter, and white matter priors for later segmentation of native images. The original T1-weighted images were segmented with the study-specific T1 template and gray matter, white matter, and CSF priors. This segmentation step involves an affine transformation of each scan to the template with a subsequent back-projection into native space. We also performed a correction for volume changes (modulation) by modu- 


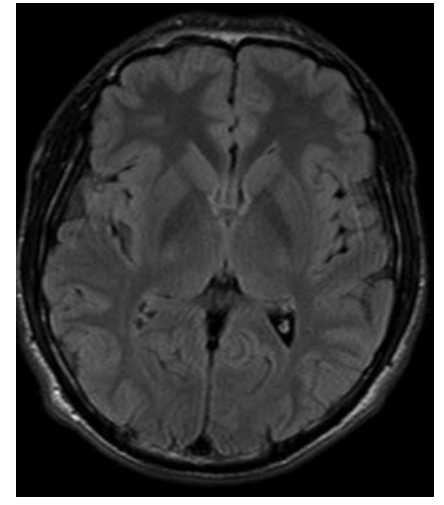

Fig 1. Axial FLAIR (TR $=9800 \mathrm{~ms}, \mathrm{TE}=110 \mathrm{~ms}$, NEX $=2$ ) image of a patient with stage 2 disease reveals normal findings.

lating each voxel by the Jacobian determinants derived from the spatial normalization. An automated brain-extraction procedure that incorporated a segmentation step was used to remove nonbrain tissue. The extracted gray and white matter images were then normalized to the specific gray and white matter templates. The normalization parameters were then applied to the original structural images in native space to reduce any contribution from nonbrain voxels and to afford optimal spatial normalization of gray matter. These normalized images were segmented into gray and white matter. Finally, the normalized and segmented images were smoothed with an 8-mm FWHM isotropic Gaussian kernel.

\section{Statistical Analysis}

The normalized modulated smoothed gray matter data were analyzed by using SPM2, within the framework of the general linear model on a voxel-wise comparison. Regional differences in gray matter volume between the patients and control subjects were assessed by using analysis of variance. Because the patients and control subjects did not show a significant difference in age and sex distribution, we did not enter these variables as covariates. The total intracranial volumes of the subjects were calculated and used as a covariate in the comparison analysis. The findings were considered significant at a voxel level of $P<.05$, corrected for multiple comparison familywise error (FWE), with an extended threshold looking for clusters with at least 20 contiguous voxels. The relationship between the clinical variables (duration of disease, immunoglobulin G [IgG] index, NDI scores) and cortical gray matter volume was investigated by regression analysis $(P<.05$, FWE-corrected). For localizing purposes, the MNI coordinates were converted to Talairach coordinates by using a dedicated script (mni2tal.m; available at: http://www.mrc-cbu.cam.ac.uk/ Imaging/Common/mnispace.shtml).

An automated software was used to retrieve the brain labels for the Talairach coordinates (http://www.talairach.org/client.html). ${ }^{14}$

\section{Results}

The differences between the mean age and sex distribution of patients and control subjects were not significant $(P>.05)$. Five of the patients were in stage 2 of the disease, and the remaining 12 patients had stage 1 SSPE. The mean clinical stage of the patients was 1.29. The mean duration of symptoms was $8.9 \pm 5.7$ weeks (range, 3-24 weeks) (Table 1). Behavioral and personality changes were present in the clinical history of all patients (100\%). The parents or teachers of 11 patients had noticed a recent decline in school performances of the pa-
Table 2: Cortical regions with decreased gray matter volume in patients compared with control subjects

\begin{tabular}{lrrrr}
\hline & \multicolumn{3}{c}{ Talairach } & \\
Anatomic Regions (BA) & Coordinates: Patients & Max. Z \\
& vs Control Subjects & Values \\
\hline & -32 & -1 & -12 & 5.49 \\
Left amygdala & -27 & 3 & -33 & 5.24 \\
Left uncus (BA 38) & -10 & -23 & 38 & 5.15 \\
Left cingulate gyrus (BA 31) & -7 & -15 & 41 & 5.32 \\
Left cingulate gyrus (BA 24) & -30 & 4 & -11 & 5.24 \\
Left superior temporal gyrus (BA 38) & -46 & -59 & -1 & 3.51 \\
Left middle temporal gyrus (BA 37) & 6 & 0 & 35 & 5.68 \\
Right cingulate gyrus (BA 24) & 52 & 3 & 13 & 5.16 \\
Right inferior frontal gyrus (BA 44) & 33 & 3 & -9 & 5.10 \\
Right superior temporal gyrus (BA 38) & 52 & -35 & -19 & 4.98 \\
Right fusiform gyrus (BA 20) & 30 & -3 & -10 & 5.13 \\
Right amygdala & & & &
\end{tabular}

Note:-Max indicates maximum; BA, Brodmann area.

tients. In addition to the behavioral changes, 5 patients also had involuntary movements in the head and limbs (myoclonic jerks). The neurologic evaluation of the patients revealed a mean NDI score of $15.8 \pm 5.3$ (range, 10-25). The findings of FLAIR and T2-weighted MR images of all patients and the control subjects were normal (Fig 1).

Voxel-based comparison analysis revealed that the patients had significantly reduced cortical gray matter volume in the frontotemporal cortical regions compared with those in the control subjects (Table 2). The regions with gray matter loss in patients included the anterior cingulate cortex (Talairach coordinates: $\mathrm{x}=-7, \mathrm{y}=-15, \mathrm{z}=41$ [Brodmann area, (BA $24)] ; x=6, y=0, z=35$ [BA 24]) and the bilateral amygdala $(\mathrm{x}=-32, \mathrm{y}=-1, \mathrm{z}=-12 ; \mathrm{x}=30, \mathrm{y}=-3, \mathrm{z}=-10)($ Fig 2$)$. The correlation analysis revealed that there was no significant association between the NDI scores and gray matter distribution of the patients. Moreover, there was no relationship between the IgG index or clinical stage and gray matter volumes of patients.

\section{Comment}

The uniformity of clinical progression in patients with SSPE suggests a common pattern of neuropathologic progress in the central nervous system. Previous autopsy reports revealed that inflammatory infiltrations in cortical gray matter are the prominent pathologic finding in the early periods of SSPE. ${ }^{1,15}$ In later stages of the disease, the neuropathologic findings spread into adjacent subcortical white matter. Early involvement of cortical gray matter would explain the nonspecific subtle behavioral symptoms that are frequently seen in the initial stages of SSPE. However, contrary to the early cortical involvement shown by the neuropathologic studies, conventional MR imaging examinations performed in patients with early disease usually reveal normal findings. Thus, cortical involvement in early SSPE could not be demonstrated in previous neuroimaging studies. The results of the current study revealed the reduced gray matter volume in the bilateral frontotemporal cortex, including the amygdala and cingulate cortex, of the patients with stage 1 and 2 SSPE.

Decreased frontotemporal cortical gray matter volume is consistent with the results of previous neuropathologic studies emphasizing the cortical involvement in the early periods of 


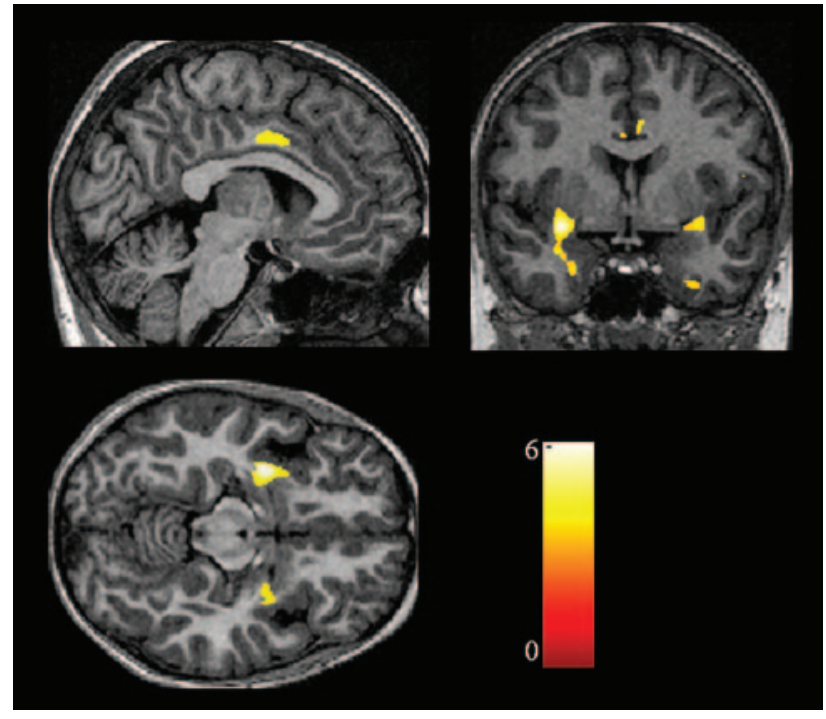

Fig 2. Statistical parametric maps demonstrating the structural difference in gray matter volumes. Significant gray matter volume decrease $(P<.05$, corrected) is overlaid in color on the normalized T1-weighted images of a healthy control subject. Only clusters of voxels consisting of at least 20 voxels are displayed. The color scale shows the range of $Z$ values. It shows the reduced gray matter volumes in bilateral medial temporal regions, including the amygdala, cingulate gyri, and right inferior frontal gyrus.

SSPE. The results of previous neuroimaging studies revealed that findings of cranial imaging performed in the initial stages of SSPE usually appear normal and that parenchymal lesions develop as the disease progresses. ${ }^{1,7}$ In the current study, we demonstrated the gray matter volume reduction in the patients with normal-appearing MR imaging findings in early stages of the disease. When we consider the results of the current and previous studies together, it suggests that a subtle loss of frontotemporal gray matter may precede the appearance of morphologic T2-weighted lesions in early SSPE. Kanemura et $\mathrm{al}^{16}$ investigated the frontal lobe volume in a patient with early SSPE. They measured the whole parenchymal volume of frontal lobes including the white and gray matters volumes. They found a decreased frontal lobe volume compared with that in healthy control subjects, which seems to be a finding consistent with the results of the current study. In another article, Kanemura and Aihara ${ }^{17}$ reported the decreased apparent diffusion coefficient in the normal-appearing frontal lobe of a patient with SSPE. They also found a correlation between the frontal diffusion changes and clinical parameters. They speculated about the possibility of an association between frontal neuropathologic changes and some of the clinical findings of SSPE.

Neurodegenerative diseases leading to progressive neuronal loss cause reduced cortical gray matter attenuation and volume in the related regions of brain. ${ }^{18}$ SSPE is caused by persistent infection of neurons and oligodendrocytes by the measles virus. Neuronal degeneration, neuronal loss, demyelination, and gliosis are the main neuropathologic findings of SSPE. The decreased gray matter volume in the frontotemporal cortex of our patients might be the consequence of neuronal degeneration/loss in those regions of brain.

The cingulate gyrus and amygdala are components of the limbic cortex, which is involved in the processing of emotions. ${ }^{19,20}$ A complex neural network, including the prefrontal and anterior cingulate gyrus, hippocampus, insular cortex, and amygdala, is involved in the processing of various aspects of emotions. The pathologies affecting the anterior cingulate gyrus and amygdala cause emotional and behavioral symptoms. The amygdala is also involved in the regulation of emotions. Thus, any pathology affecting the amygdala may cause dysregulation of emotional processing leading to over- or underexpression of emotions. The initial symptoms of patients with SSPE include emotional instability, inappropriate emotions, inappropriate expression of emotions, and other behavioral changes. Cortical involvement in the cingulate gyrus and amygdala may explain the emotional and behavioral symptoms of early SSPE.

Cognitive decline, which is usually recognized as a recent decrease in scholastic performance of the patient, is another common symptom of early SSPE. The anterior cingulate cortex together with the orbitofrontal cortex takes part in the processing of decision making. ${ }^{21}$ The amygdala is also involved in reward-based learning and decision making. ${ }^{22}$ Recent studies have shown that the amygdala is a critical structure in a neural system necessary for implementing advantageous decisions. Thus, the gray matter volume reduction in the anterior cingulate cortex and the amygdala may contribute to the appearance of cognitive decline during the initial stages of SSPE. However, we did not find a significant correlation between the NDI scores of patients and their cortical gray matter distribution. The NDI scoring is a gross evaluation of the motor and sensory functions as well as the mental states of patients with SSPE. The lack of a correlation between the NDI scores and gray matter volume might be because we studied only patients in the initial stages of the disease, and their NDI scores were distributed in a narrow range.

The correlation analysis in the current study did not reveal any association between the duration of symptoms and gray matter distribution in patients. This finding suggests that the gray matter loss in the frontotemporal regions might have developed at very early periods of the disease. The relatively narrow range of duration of symptoms among patients might be another explanation of this finding. Furthermore, we did not find any correlation between the IgG index and the gray matter distribution. This finding indicates that there is no association between the degree of neuroimmunologic reaction and gray matter loss in early SSPE.

Several quantitative imaging studies with a region-of-interest design such as quantitative diffusion MR imaging and proton MR spectroscopy ${ }^{8,9,17}$ have been conducted to investigate the in vivo neuropathologic findings in patients with early SSPE. Because conventional imaging findings usually appear normal in early SSPE, quantitative imaging studies investigating patients with early SSPE require a prior hypothesis of localization. The frontotemporal weighting of cortical gray matter loss demonstrated by the current study may guide future studies investigating early imaging findings of SSPE.

A few limitations of the current study should be mentioned. First, because of the ethical issues, we did not perform a longitudinal study. A longitudinal study would give valuable information about the evolution of gray matter loss during the clinical progression of the disease. The second limitation may be that we could not measure the severity of the patients' emotional symptoms. Thus, we could not investigate the correla- 
tion between volumetric changes in gray matter and severity of behavioral symptoms. Although the comparison analysis revealed a gray matter reduction in the emotion-related regions of the brain, a correlation analysis would be helpful to investigate the association of the severity of emotional symptoms and gray matter distribution.

In conclusion, the results of the current study demonstrated the decreased gray matter volume in the frontotemporal cortex of patients in the early stages of SSPE. The gray matter volume reduction in the cingulate gyrus and amygdala, which are related to decision making and emotion processing, may contribute to the development of behavioral symptoms of early SSPE.

\section{References}

1. Garg RK. Subacute sclerosing panencephalitis. Postgrad Med J 2002;78:63-70

2. Yalaz K, Anlar B, Renda Y, et al. Subacute sclerosing panencephalitis in Turkey: epidemiological features. J Trop Pediatr 1988;34:301-05

3. Brismar J, Generoso GG, Steyern KV, et al. Subacute sclerosing panencephalitis: evaluation with CT and MR. AJNR Am J Neuroradiol 1996;17:761-72

4. Anlar B, Saatci I, Köse G, et al. MRI findings in subacute sclerosing panencephalitis. Neurology 1996;47:1278-83

5. Akdal G, Baklan B, Cakmakci H, et al. MRI follow-up of basal ganglia involvement in subacute sclerosing panencephalitis. Pediatr Neurol 2001;24:393-95

6. Tuncay R, Akman-Demir G, Gökyigit A, et al. MRI in subacute sclerosing panencephalitis. Neuroradiology 1996;38:636-40

7. Ozturk A, Gurses C, Baykan B, et al. Subacute sclerosing panencephalitis: clinical and magnetic resonance imaging evaluation of 36 patients. J Child Neurol 2002;17:25-29
8. Aydin K, Tatli B, Ozkan M, et al. Quantification of neurometabolites in subacute sclerosing panencephalitis by 1H-MRS. Neurology 2006;67:911-13

9. Alkan A, Saral K, Kutlu R, et al. Early- and late-state subacute sclerosing panencephalitis: chemical shift imaging and single-voxel MR spectroscopy AJNR Am J Neuradiol 2003;24:501-06

10. Ashburner J, Friston KJ. Voxel-based morphometry: the methods. Neuroimage 2000;11:805-21

11. Jabbour JT, Garcia JH, Lemmi H, et al. Subacute sclerosing panencephalitis: a multidisciplinary study of eight cases. JAMA 1969;207:2248-54

12. Dyken PR, Swift A, Durant RH. Long-term follow-up of patients with subacute sclerosing panencephalitis treated with inosiplex. Ann Neurol 1982;11:359-64

13. Good CD, Johnsrude IS, Ashburner J, et al. A voxel-based morphometric study of ageing in $\mathbf{4 6 5}$ normal adult human brains. Neuroimage 2001;14:21-36

14. Lancaster JL, Woldorff MG, Parsons LM, et al. Automated Talairach atlas labels for functional brain mapping. Hum Brain Mapp 2001;10:121-31

15. Oya T, Martinez AJ, Jabbour JT, et al. Subacute sclerosing panencephalitis: correlation of clinical, neurophysiologic and neuropathological findings. Neurology 1974;24:211-18

16. Kanemura H, Aihara M, Okubo T, et al. Sequential 3-D MRI frontal volume changes in subacute sclerosing panencephalitis. Brain Dev 2005;27:148-51

17. Kanemura $H$, Aihara M. Serial diffusion-weighted imaging in subacute sclerosing panencephalitis. Pediatr Neurol 2008;38:430-34

18. Frisoni GB, Testa C, Zorzan A, et al. Detection of grey matter loss in mild Alzheimer's disease with voxel based morphometry. J Neurol Neurosurg Psychiatry 2002;73:657-64

19. Davidson RJ, Putnam KM, Larson CL. Dysfunction in the neural circuitry of emotion regulation: a possible prelude to violence. Science 2000;289:591-94

20. Cahill L, Haier RJ, Fallon J, et al. Amygdala activity at encoding correlated with long-term, free recall of emotional information. Proc Natl Acad Sci U S A 1996;93:8016-21

21. MW Cole, Schneider W. The cognitive control network: integrated cortical regions with dissociable functions. Neuroimage 2007;37:343-60

22. Bechara A, Damasio H, Damasio AR, et al. Different contributions of the human amygdala and ventromedial prefrontal cortex to decision-making. $\mathrm{JNeu}$ rosci 1999;19:5473-81 